\title{
A Study on the Tyrosinase Inhibitory and Antioxidant Effect of Microalgae Extracts
}

\author{
Keunho $\mathrm{Ji}^{1}$, Yeeun $\mathrm{Kim}^{2}$, and Young Tae $\mathrm{Kim}^{2} *$ \\ ${ }^{1}$ Basic Science Research Institute, ${ }^{2}$ Deptartment of Microbiology, Pukyong National University, Busan 48513, Republic of Korea
}

Received: December 7, 2020 / Revised: February 16, 2021 / Accepted: February 26, 2021

\begin{abstract}
Reactive oxygen species (ROS) disrupt the cellular redox balance, exert cytotoxic effects, and consequently promote the development of various diseases in humans. Previous studies have reported that antioxidants counteract the adverse effects of ROS. Several studies examine the whitening effects of various agents based on their ability to inhibit tyrosinase activity. Tyrosinase is a critical enzyme involved in the synthesis of melanin, which protects the skin against radiation. Various agents exhibiting antioxidant and tyrosinase inhibitory activities have been synthesized. However, these synthetic drugs are associated with toxicity, decreased safety, and poor skin penetration in vivo, which has limited the clinical application of synthetic drugs. This study examined the antioxidant and tyrosinase inhibitory activities of some microalgae. The methanol, dichloromethane, and ethyl acetate extracts of four microalgal species (Tetraselmis tetrathele, Dunaliella tertiolecta, Platymonas sp., and Chaetoceros simplex) were prepared. The physiological and whitening effects of microalgal extracts were investigated by measuring the antioxidant and tyrosinase inhibitory activities. The ethyl acetate extract of $D$. tertiolecta exhibited the highest antioxidant and tyrosinase inhibitory activities. Future studies must focus on examining the whitening effects of microalgae on cell lines to facilitate the development of microalga-based therapeutics for skin diseases, functional health foods, and whitening agents. Thus, microalgae have potential applications in the pharmaceutical, food, and cosmetic industries.
\end{abstract}

Keywords: Antioxidant, cosmeceutical, Dunaliella, microalgae, tyrosinase inhibition

\section{Introduction}

The pro-oxidant and antioxidant balance in the human body can bust because of physical or environmental factors. The reactive oxygen species (ROS), one kind of direct causative at oxidative stress, has induced oxidation. It rises to cell damage and a pathological disease like cancer [1]. These defense mechanisms against oxidative stress called the anti-oxidation are the mechanism for inhibiting the production or returned to normal levels that the over-produced free radicals at the energy production process in vivo [2]. Reactive oxygen species

\section{* Corresponding author}

Tel: +82-51-629-5616, Fax: +82-51-629-5619

E-mail: ytkim@pknu.ac.kr are produced in the process of stabilizing by taking incomplete electrons generated during the mitochondrial respiratory process from nearby molecules, and types include singlet oxygen $\left({ }^{1} \mathrm{O}_{2}\right)$, superoxide $\left(\mathrm{O}^{2-}\right)$, hydroxyl radical $(\mathrm{OH})$, and hydrogen peroxide $\left(\mathrm{H}_{2} \mathrm{O}_{2}\right)[2$, 3]. These reactive oxygen species are very unstable and highly reactive, easily react with various biological materials, and attack the polymers in the body, causing irreversible damage to cells and tissues, mutations, and cancer. It is also known to cause diabetes, brain disease, and cardiovascular disease. Therefore, for the prevention and treatment of many chronic degenerative diseases in which oxidative stress caused by free radicals acts as a risk factor, many studies have been conducted to find antioxidants from natural products with low toxicity and side effects [3]. 
Melanin is essential to protect human skin from radiation, and recent studies have shown that it also plays an important role in regulating skin homeostasis. However, abnormal melanin accumulation causes pigment abnormalities such as blemishes, melanoma, freckles, senile black spots, and damages internal tissues, and causes skin aging due to light and skin quality. It also causes enzymatic browning in fruits and vegetables. Melanin is the final product produced by melanogenesis and is influenced by various factors such as hormonal changes and nutritional status. Besides, melanogenesis can cause damage to cell membranes and DNA by generating free radicals, causing DNA lipid peroxidation, reducing membrane fluidity, and DNA mutations leading to cancer and degenerative diseases $[4,5]$. This melanogenesis occurs in melanocytes located in the epidermis basement layer, and tyrosinase acts as the main enzyme in this process. Tyrosinase, also called polyphenol oxidase (PPO), is a monooxygenase containing copper and a major enzyme in melanin synthesis, widely distributed in microorganisms, animals, and plants. Tyrosinase catalyzes two oxidation reactions that hydroxylate Ltyrosine to convert to L-dihydroxyphenylalanine (DOPA) and oxidize L-DOPA to DOPA quinone. Highly reactive DOPA quinone reacts with amino acids and proteins to form high molecular compounds or brown pigments (eumelanin or pheomelanin). It is possible to develop a functional cosmetic or a therapeutic agent to treat pigment disorders as a whitening agent by inhibiting melanin production by inhibiting tyrosinase activity. To date, several tyrosinase inhibitors have been synthesized and isolated to inhibit tyrosinase activity. Still, the use of existing drugs has been limited due to their toxicity, low safety, and insufficient skin penetration and activity. Therefore, efforts have been made to find new tyrosinase inhibitors in natural products to reduce the side effects of synthetic tyrosinase inhibitors [6].

Recently, marine resources are attracting attention as raw materials for bioactive compounds to develop new drugs and healthy foods. Among them, marine microalgae are creatures in which more than 50,000 species are distributed in various environments, and they provide various biochemical substances with vast biodiversity. Microalgae are rich in essential trace elements such as vitamins, minerals, iodine, zinc, and various functional polysaccharides and polyphenols, and secondary carot- enoids such as eicosapentaenoic (EPA) and docosahexaenoic (DHA) acids and long-chain polyunsaturated fatty acids (PUFA). It is known that metabolites are also produced. Also, research is being conducted to use as a renewable biofuel using microalgae. Microalgae are single-celled organisms with vast biodiversity and are capable of rapidly growing under a large-scale system that is easy to produce large amounts of biomass and can continuously supply desire molecules. Besides, bulk microalgal biomass or fractions thereof can be used for nutritional applications [7, 8]. Therefore, in this study, microalgae, which is an eco-friendly material, was selected as a sample for removing free radicals and inhibiting tyrosinase.

Therefore, this study aimed to investigate the antioxidant activity capable of removing free radical species and the tyrosinase inhibitory activity that can inhibit melanin production by using the microalgae extract, which is a natural product. Through this, it finds microalgae extracts that have both antioxidant and tyrosinase inhibitory activities and provides useful information for researching treatments for skin diseases related to chronic degenerative diseases and pigment disorders caused by oxidative stress and developing health supplements. As such, it is considered that it can be used as a raw material for functional cosmetics.

\section{Materials and Methods}

\section{Microalgae}

Microalgae were provided and used by the South Sea Fisheries Research Institute of NIFS (National Institute of Fisheries Science). Microalgae were studied by selecting species whose antioxidant activity and whitening activity was not verified. Three green algae species (Tetraselmis tetrathele, Dunaliella tertiolecta, Platymonas sp.) and one diatom species (Chaetoceros simplex) were used. All algal cells were cultured in Conwy medium at $25^{\circ} \mathrm{C}$ for $1-2$ weeks under continuous lighting conditions.

\section{Microalgal extracts preparation}

For obtained the active substance from microalgae, we performed the extraction using an organic solvent. The microalgal culture was centrifuged at 8,000 rpm for $30 \mathrm{~min}$ to obtain $10 \mathrm{~g}$ of microalgal cells. $50 \mathrm{ml}$ of methanol was added to the microalgal cells and performed 


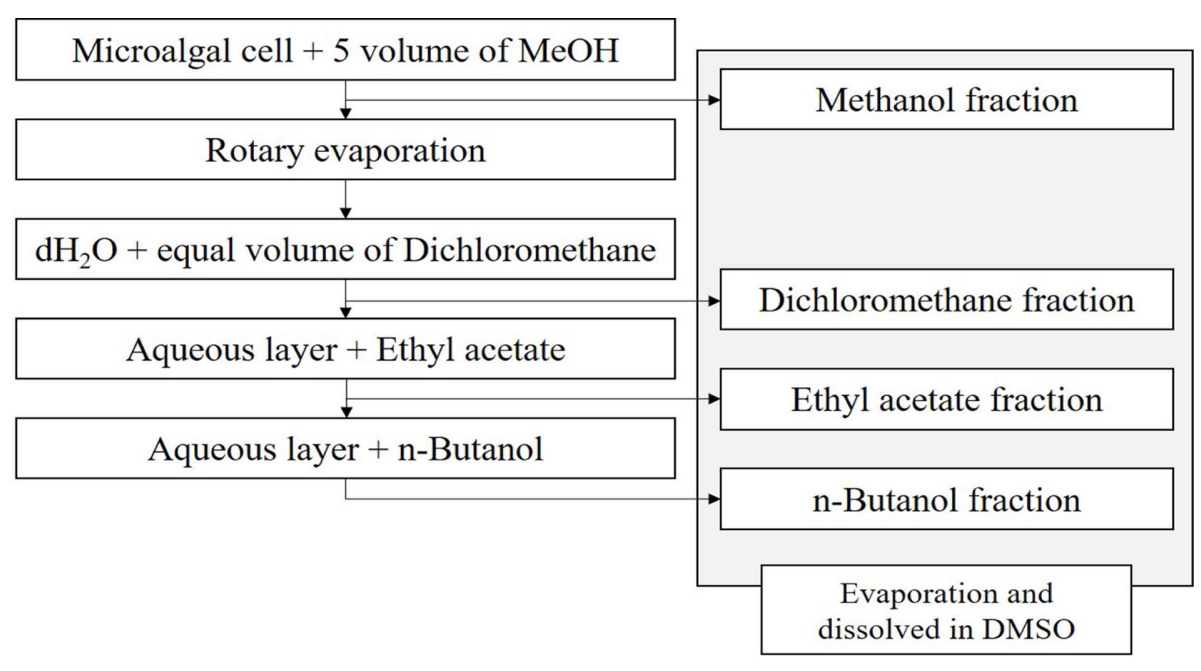

Fig. 1. The extraction process using organic solvent.

extraction at $25^{\circ} \mathrm{C}, 150 \mathrm{rpm}$ for $48 \mathrm{~h}$. Then extracts were concentrated on a rotary evaporator. $100 \mathrm{ml}$ of sterile distilled water and $100 \mathrm{ml}$ of dichloromethane were added in methanol extract and performed extraction at $25^{\circ} \mathrm{C}, 150 \mathrm{rpm}$ for $48 \mathrm{~h}$. In the same way, ethyl acetate fraction was obtained. The butanol fraction was obtained using butanol in the same way. Each obtained solvent fraction layer was concentrated on a rotary evaporator, and then dissolved in DMSO reach at $10 \mu \mathrm{g} / \mathrm{ml}$. Remain the aqueous layer was used as $\mathrm{H}_{2} \mathrm{O}$ extract after evaporation of the solvent (Fig. 1).

\section{Total polyphenol contents}

For the measurement of total polyphenol contents according to extraction solvents, $10 \mu \mathrm{l}$ of sample and $200 \mu \mathrm{l}$ of $2 \% \mathrm{Na}_{2} \mathrm{CO}_{3}$ were added in 96 well microplates. Next, a reaction for $3 \mathrm{~min}$ at room temperature, and then, a reaction for $30 \mathrm{~min}$ adding $10 \mu \mathrm{l}$ of $50 \%$ Folin solution. Done to reaction, optical density was measured at $700 \mathrm{~nm}$ using a microplate reader and calculates of total polyphenol contents in the following equation.

$$
\begin{aligned}
& \text { Total polyphenol contents }(\%)= \\
& \quad(\text { control OD }- \text { sample OD }) / \text { control OD } \times 100
\end{aligned}
$$

\section{DPPH radical scavenging activity}

To measure the DPPH radical scavenging activity according to extraction solvents, $25 \mu \mathrm{l}$ of sample and $75 \mu \mathrm{l}$ of $0.2 \mathrm{mM}$ DPPH solution were added in 96 well microplates and reaction for $30 \mathrm{~min}$ at dark condition. After the reaction, optical density was measured at $517 \mathrm{~nm}$ by a microplate reader. Ascorbic acid $(1 \mathrm{mg} / \mathrm{ml})$ was used to positive control and calculate the following equation.

$$
\begin{aligned}
& \text { DPPH radical scavenging activity }(\%)= \\
& \quad(\text { control OD }- \text { sample OD)/control OD } \times 100
\end{aligned}
$$

\section{ABTS radical scavenging activity}

The ABTS radical scavenging activity was measured. In a 96 well microplate, $50 \mu \mathrm{l}$ of sample and $50 \mu \mathrm{l}$ of ABTS solution were added and reacted for $30 \mathrm{~min}$ at dark conditions. After the reaction, measuring the absorbance at $734 \mathrm{~nm}$ in the microplate reader. The ABTS solution was used after reaction at $24 \mathrm{~h}$ made of one by one mixture as $7.4 \mathrm{mM}$ ABTS and $2.6 \mathrm{mM}$ potassium persulfate. Ascorbic acid ( $1 \mathrm{mg} / \mathrm{ml})$ was used to positive control and calculate the following equation.

$$
\begin{aligned}
& \text { ABTS radical scavenging activity }(\%)= \\
& \quad(\text { control OD }- \text { sample OD)/control OD } \times 100
\end{aligned}
$$

\section{The measure of tyrosinase inhibition activity}

The $110 \mu \mathrm{l}$ of $0.1 \mathrm{M}$ sodium phosphate buffer ( $\mathrm{pH}$ 6.5) and $10 \mu \mathrm{l}$ of mushroom tyrosinase $(500 \mathrm{U} / \mathrm{ml})$ were mixed in order loaded into 96 well microplates. The $20 \mu \mathrm{l}$ of tyrosine was adding to that mixture and reaction for $15 \mathrm{~min}$ at $37^{\circ} \mathrm{C}$. The reaction was finished in ice for $5 \mathrm{~min}$, and then absorbance was measured at $475 \mathrm{~nm}$ 
using a microplate reader. The positive control was used of ascorbic acid $(1 \mathrm{mg} / \mathrm{ml})$. The calculate the following equation.

$$
\begin{aligned}
& \text { Tyrosinase inhibition activity }(\%)= \\
& (\text { control OD }- \text { sample OD)/control OD } \times 100
\end{aligned}
$$

In each item's calculation formula, the control OD was calculated by adding the same amount of water instead of the extract. Also, the resulting figure was converted into a relative value for the result of $1 \mathrm{mg} / \mathrm{ml}$ ascorbic acid used as a positive control group and is schematically illustrated.

\section{Statistics processing}

All experiments were repeated three times. Statistical processing was calculated as mean \pm S.D for each sample, and the significance was verified by calculating the $\mathrm{p}$-value.

\section{Results and Discussion}

It is known that there are more than 100,000 kinds of microalgae worldwide, and some of them have been confirmed to function. Active research is being conducted in fields such as energy, industrial material production, and greenhouse gas reduction. In particular, major companies in the world invest in new and renewable energy and are making efforts to secure technology. Among the



Fig. 2. Total polyphenol contents of microalgal extracts. fields where the use of microalgae is likely to be expanded is the chemical field. Currently, the food sector industry using Chlorella and Spirulina is the most active [9], but it is expected to expand into fields such as pharmaceuticals, cosmetic raw materials, and bioplastics [10].

The standard substance in the measurement of total polyphenol content was using tannic acid. The results in measured tannic acid equivalent polyphenol contents, the best one was ethyl acetate extract fraction of $D$. tertiolecta. The content was $26 \mathrm{mg} / 100 \mathrm{~g}$ polyphenol. The next one was the dichloromethane extract fraction of D. tertiolecta $(16 \mathrm{mg} / 100 \mathrm{~g})$. Besides, at the aqueous layer of $D$. tertiolecta and the ethyl acetate extract fraction of Platymonas sp. were observed $13 \mathrm{mg} / 100 \mathrm{~g}$ of polyphenol contents (Fig. 2). The result was not described in the butanol fraction because no activity was observed in all measurement items. The DPPH radical scavenging occurred only ethyl acetate extract fraction. The scavenging activities were $39.05 \%, 15.29 \%, 10.33 \%$, and $8.06 \%$ in order D. tertiolecta, C. simplex, Platymonas sp. and T. tetrathele, respectively (Fig. 3). The highest value of ABTS radical scavenging activity was $89.77 \%$ at ethyl acetate extract fraction of $D$. tertiolecta. In sequentially, ethyl acetate extract fraction and dichloromethane extract fraction of C. simplex were showed $62.67 \%$ and $58.27 \%$. The dichloromethane extract fraction of $D$. tertiolecta, was showed 57.18\% results in the ABTS scavenging activity (Fig. 4). The difference in ABTS and

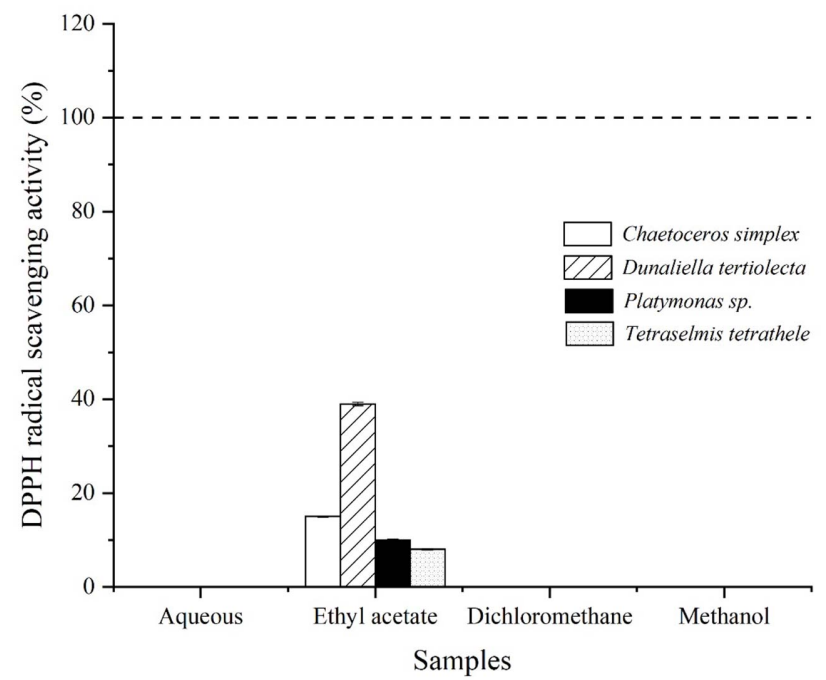

Fig. 3. DPPH radical scavenging activity of macroalgal extracts. 


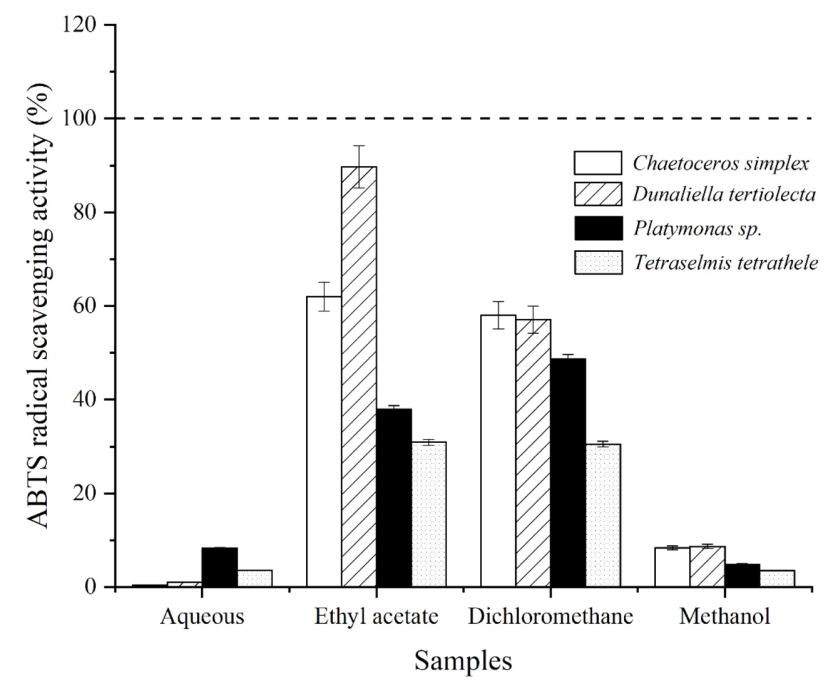

Fig. 4. ABTS radical scavenging activity of macroalgal extracts.

DPPH radical scavenging activity according to the extraction solvent is expected to be due to the difference in the binding capacity of cation radical and free radical. This study investigated the possibility of development as a therapeutic agent for skin diseases, health functional foods, and whitening agents using four types of microalgae and extracted antioxidants using organic solvents to measure antioxidant and tyrosinase inhibitory activities. As a result of the antioxidant activity test, the best activity was found in the ethyl acetate extract of $D$. tertiolecta. The dichloromethane extract of $D$. tertiolecta and the ethyl acetate extract of $C$. simplex also showed relatively good antioxidant activity. Besides, some antioxidant activity was also observed in ethyl acetate extract of Platymonas sp. According to a study by Wang et al., it has been reported that total polyphenol compounds are soluble in polar organic solvents such as acetone [11]. Another study reported that ethyl acetate could be a better solvent for polyphenol extraction than water [12]. As a result of this study, when comparing the antioxidant activity according to the organic solvent, ethyl acetate extract and dichloromethane extract showed high antioxidant activity. It is thought that the extraction efficiency was high in the polar organic solvent as the results of the previous researchers.

The tyrosinase inhibitory activity according to the microalgae extract was highest at $124 \%$ in ethyl acetate extract of Platymonas sp. Moreover, observed 107\% and

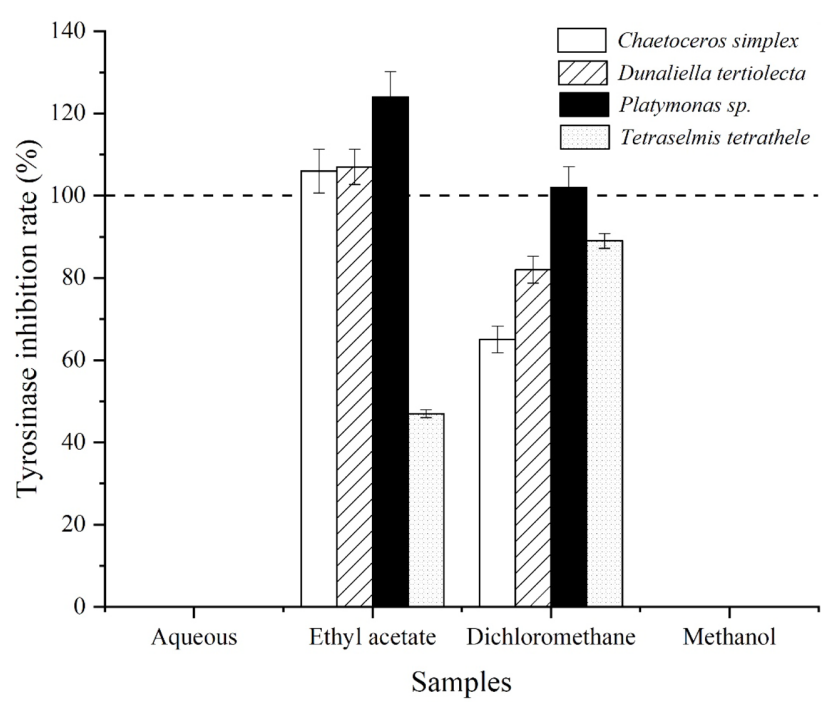

Fig. 5. Tyrosinase inhibition activity of algal extracts.

$106 \%$ in ethyl acetate extract of $D$. tertiolecta and $C$. simplex. Also, dichloromethane extract of Platymonas sp. was $102 \%$. Also, could confirm that the inhibition activity of the $89 \%, 82 \%$, and $65 \%$ in dichloromethane extract on $T$. tetrathele, D. tertiolecta, and C. simplex, respectively (Fig. 5). As a result of tyrosinase inhibitory activity, ethyl acetate extract of Platymonas sp. showed the best activity, and ethyl acetate extract of $D$. tertiolecta and C. simplex, and dichloromethane extract of Platymonas sp. showed relatively good inhibitory activity. The tyrosinase inhibitory activity was found in ethyl acetate and dichloromethane fraction, but not in the aqueous layer and methanol fraction. Therefore, the active material is expected to have an intermediate polarity. Since they are natural products that have similar ascorbic acid activity and are harmless to the human body, they are considered competitive whitening active substances as their substitutes compared to chemical compounds. When comparing tyrosinase inhibitory activity according to the organic solvent, ethyl acetate extract and dichloromethane extract showed high antioxidant activity.

Among the microalgae used in this study, Dunaliella is known to biosynthesize carotenoid pigments such as $\alpha$-, $\beta$-carotene, violaxanthin, neoxanthin, zeaxanthin, and lutein [13]. Also, the use of glycerol biosynthesis, wastewater treatment, and SCP has already been studied [14]. However, D. tertiolecta has been studied for 
antihypertensive, bronchodilator, antiseritonin, polysynaptic block, analgesic, muscle relaxant, and antioedema activities [15-17], but no results have been reported for antioxidant and whitening activities. A study on the antioxidant activity of $C$. simplex can be found in a report by Karthikeyan et al. [18] about $55.69 \%$ of DPPH radical scavenging activity was confirmed in acetone extract. As a result of the antioxidant activity measurement of $T$. tetrathele, there is a study report that it has an activity of $2.99 \mathrm{mg} \mathrm{GAE} / \mathrm{g}$ [19]. As described above, the antioxidant activity of the microalgae used in this study has already been revealed, but this study has a difference as a study that reveals that it has both antioxidant and whitening activity and is further used in product development utilizing the results of this study. It is believed to have provided us with useful information and secured better usability. Abd-El-Baky et al. reported that when microalgae were cultured in a stressful environment, the accumulation of antioxidants increased [20]. Also, the Nannochloropsis oculata improvement strain, which enhanced the EPA content, obtained by NMU (N-nitroso-N-methylurea) and quizalofopp-ethyl treatment [21], and the Arthrospira platensis improvement strain, which improved $\mathrm{CO}_{2}$ fixation through EMS (ethyl methane sulfate) are also reported [22]. To increase the antioxidant and whitening activity of the microalgae used in this study, it is expected that positive and effective utilization in the healthy functional food and cosmetics field is possible if further studies are conducted based on the previous research.

\section{Acknowledgments}

This work was supported by the Research Grant of Pukyong National University (year 2019).

\section{Conflict of Interest}

The authors have no financial conflicts of interest to declare.

\section{References}

1. Koh YJ, Cha DS, Choi HD, Park YK, Choi IW. 2008. Hot water extraction optimization of dandelion leaves to increase antioxidant activity. Korean J. Food Sci. Technol. 40: 283-289.

2. Seo BY, Choi MJ, Choi EA, Park E. 2014. In vitro antioxidant effects of Sarijang. J. Korean Soc. Food Sic. Nut. 43: 618-623.
3. Choi SY, Kim JH, Lee J, Cho EJ. 2017. Protective effect of Acer okamotoanum from oxidative stress in $\mathrm{C} 6$ glial cells. J. Appl. Biol. Chem. 60: 141-147.

4. Kwak JY, Seok JK, Suh HJ, Hong SS, Kim DS, Boo YC. 2016. Antimelanogenic effects of luteolin 7-sulfate isolated from Phyllospadix iwatensis Makino. Trans. Res. 50: 501-511.

5. Ji K, Cho YS, Kim YT. 2018. Tyrosinase inhibitory and anti-oxidative effects of lactic acid bacteria isolated from dairy cow feces. Probiotics Antimicrob. Proteins 10: 43-55.

6. Yoon NY, Eom TK, Kim MM, Kim SK. 2009. Inhibitory effect of phlorotannins isolated from Ecklonia cava on mushroom tyrosinase activity and melanin formation in mouse B16F10 melanoma cells. J. Agric. Food Chem. 57: 4124-4129.

7. Cha SH, Ko SC, Kim D, Jeon YJ. 2011. Screening of marine algae for potential tyrosinase inhibitor: those inhibitors reduced tyrosinase activity and melanin synthesis in zebrafish. J. Dermatol. 38: 354-363.

8. Pereira H, Custodio L, Rodrigues MJ, Sousa Bd, Oliveira M, Barreira L, et al. 2015. Biological activities and chemical composition of methanolic extracts of selected autochthonous microalgae strains from the Red Sea. Mar. Drugs 13: 3531-3549.

9. Andrade LM, Andrade CJ, Dias M, Nascimento CAO, Mendes MA. 2018. Chlorella and spirulina microalgae as sources of functional doods, nutraceuticals and food supplements; an overview. MOJ Food Process Technol. 6: 45-58.

10. Rizwan M, Mujtaba G, Memon SA, Lee K, Rashid N. 2018. Exploring the potential of microalgae for new biotechnology applications and beyond: a review. Renew. Sustain. Energy Rev. 92: 394404.

11. Wang T, Jonsdottir R, Ólafsdóttir G. 2009. Total phenolic compounds, radical scavenging and metal chelation of extracts from Icelandic seaweeds. Food Chem. 116: 240-248.

12. Morowvat MH, Ghasemi Y. 2016. Evaluation of antioxidant properties of some naturally isolated microalgae: Identification and characterization of the most efficient strain. Biocata. Agric. Biotechnol. 8: 263-269.

13. Ben-Amotz A. 1995. New mode of Dunaliella biotechnology: two-phase growth for $\beta$-carotene production. J. Appl. Phycol. 7: 65-68.

14. Hosseini Tafreshi A, Shariati M. 2009. Dunaliella biotechnology: methods and applications. J. Appl. Microbiol. 107: 14-35.

15. Borowitzka MA. 1995. Microalgae as sources of pharmaceuticals and other biologically active compounds. J. Appl. Phycol. 7: 3-15.

16. Villar R, Laguna MR, Calleja JM, Cadavid I. 1992. Effects of Phaeodactylum tricornutum and Dunaliella tertiolecta extracts on the central nervous system. Planta Med. 58: 405-409.

17. Chang T, Ohta S, Ikegami N, Miyata H, Kashimoto T, Kondo M. 1993. Antibiotic substances produced by a marine green alga, Dunaliella primolecta. Bioresour. Technol. 44: 149-153.

18. Karthikeyan $P$, Manimaran K, Sampathkumar $P$, Jaikumar $M$, Robin RS, Saravanakumar C, et al. 2013. In vitro antioxidant activity of marine diatoms. J. Environ. Sci. Toxicol. Food Technol. 5: 32-37.

19. Farahin AW, Yusoff FM, Nagao N, Basri M, Shariff M. 2016. Pheno- 
lic content and antioxidant activity of Tetraselmis tetrathele (West) Butcher 1959 cultured in annular photobioreactor. J. Environ. Biol. 37: 631-639.

20. Abd El-Baky HH, Hussein MM, El-Baroty GS. 2008. Algal extracts improve antioxidant defense abilities and salt tolerance of wheat plant irrigated with sea water. Afr. J. Biochem. Res. 2: 151-164.

21. Yoo C, Kim CJ, Choi GG, Ahn CY, Choi JS, Oh HM. 2009. A mutant
Arthrospira platensis M20CJK3 showing enhanced growth rate and floatation activity. Korean J. Microbiol. 45: 268-274.

22. Cordero BF, Obraztsova I, Couso I, Leon R, Vargas MA, Rodriguez H. 2011. Enhancement of lutein production in Chlorella sorokiniana (Chorophyta) by improvement of culture conditions and random mutagenesis. Mar. Drugs 9: 1607-1624. 